\title{
EL RUIDO QUE SE ESCAPA: LO NO IDIOMÁTICO COMO POSICIONAMIENTO CRÍTICO ANTE EL PROCESO DE NORMATIVIZACIÓN DE LA MÚSICA IMPROVISADA
}

\author{
Héctor Rey Vizcaíno \\ Universidad del País Vasco / Euskal Herriko Unibertsitatea, Dpto. Escultura \\ Doctorando
}

\section{Resumen}

En esta disertación se expone una revisión de la noción de improvisación no idiomática. Introducida por el músico británico Derek Bailey, ganó notoriedad gracias a su libro La Improvisación: su naturaleza y su práctica en la música, publicado en 1980. La música improvisada, a pesar de su celebración de la fugacidad e irrevocabilidad del momento presente, no está exenta de influencias que la dirigen hacia su asentamiento y cristalización. Este artículo se ocupa de caracterizar la agencia de estas fuerzas con la intención de fundar un entendimiento de lo no idiomático como la posibilidad de la praxis musical improvisada de adoptar un posicionamiento crítico frente a los procesos de subjetivación que la persiguen.

Palabras clave: MÚSICA; RUIDO; ESCUCHA; IMPROVISACIÓN; IDIOMA

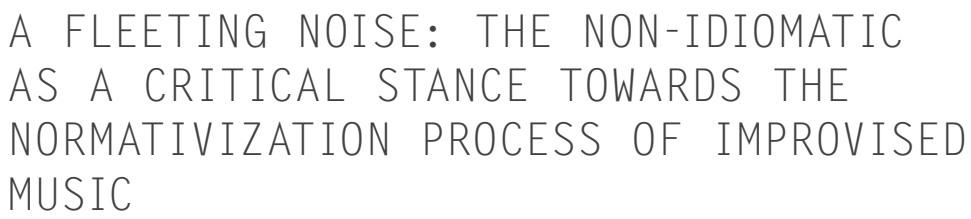

Abstract

This paper expounds a critical review of the notion of non-idiomatic improvisation. First introduced by the English musician Derek Bailey, it gained popularity with the release of his 1980 book Improvisation: its Nature and Practice in Music. Improvised music, in spite of its celebration of the fleeting nature and irrevocability of the present moment, is also subjected to influences leading to its settlement and stagnation. This article will describe the agency of those forces with the aim of establishing an understanding of the non-idiomatic not as a specific musical formulation but as the possibility of improvised musical praxis to take a critical stance towards the processes of subjectivation trying to chase it down.

Keywords: MUSIC; NOISE; LISTENING; IMPROVISATION; IDIOM

\footnotetext{
Rey Vizcaíno, Héctor. 2015. "El ruido que se escapa: 10 no idiomático como posicionamiento crítico ante el proceso de normativización de la música improvisada". AusArt 3 (2): 83-97. DOI: 10.1387/ausart.15485
}

\section{AUSART}




\section{INTRODUCCIÓN: LA NATURALEZA ELUSIVA DE LA IMPROVISACIÓN LIBRE}

La improvisación libre es una manera de hacer música surgida a mediados de los años sesenta del siglo XX en el Reino Unido cuya especificidad reside en renunciar deliberadamente a toda prefiguración composicional que condicione a priori la articulación de los vectores sonoros. Esto impone en los músicos la responsabilidad de calibrar el flujo musical en tiempo real a través de la puesta en común y la negociación de sus intereses y aspiraciones estéticas. Una música de etos antiteleológico que, al enfatizar la atención hacia el momento presente y sus contingencias, parece contemplar la posibilidad de sortear toda categorización genérica, situándose al margen de los idiomas musicales establecidos.

No obstante, su capacidad de deslastrar completamente toda influencia idiomática ha sido igualmente defendida y discutida por diferentes generaciones de practicantes y estudiosos de la música libremente improvisada. Durante más de medio siglo de historia se han ido conformando procesos de codificación que han devenido en modelos canónicos que, a su vez, han sido cuestionados y contestados.

Podemos entender este carácter dialéctico que surge ante el agotamiento de los lenguajes si atendemos a un fenómeno característico de la historia de la música: la tensión entre escucha y ruido. El ruido, continuo presubjetivo de todo lo audible, de naturaleza eminentemente elusiva, está siempre a la fuga de los mecanismos de construcción subjetiva de la escucha y su poder de dar forma y sentido. Así, por ejemplo, el dodecafonismo en el siglo XX, al ampliar la extensión de la legalidad armónica hacia nuevos límites, termina con la noción de ruido armónico. De la misma manera, toda la música desde Luigi Russolo hasta el 4'33" de Cage ha configurado una sucesión de procesos de asentamiento y normalización que han sido respondidos por discontinuidades y cambios de paradigma ante el agotamiento de los lenguajes. La improvisación libre, por su parte, no ha sido inerte a estos procesos.

La finalidad de este artículo es identificar y caracterizar la continuidad y recurrencia, en la música libremente improvisada, de una potencia contraria a todo proceso de normativización, una fuerza que apunta siempre hacia un afuera inalcanzable para la subjetividad, y reconocer en ésta su potencial no idiomático. 


\section{NO-IDIOMA}

La noción de improvisación no idiomática aparece por primera vez en el libro Improvisation: its Nature and Practice in Music, escrito por Derek Bailey en 1980. Preocupado por la naturaleza de la improvisación, Bailey se pregunta qué tiene de específica su manera de improvisar, por aquel entonces ya tan alejada de las convenciones del jazz que habían condicionado su aprendizaje musical. En su introducción, el músico británico advierte de que, en el libro, el uso del término 'improvisación no idiomática' responderá a una diferencia esencial entre el sentido y función que adquiere el acto de improvisar en la denominada libre improvisación y el que adquiere en otras músicas que incluyen la improvisación -como en la música barroca, el jazz o las músicas de tradición popular de diferentes culturas-. Según Bailey, en estas últimas la improvisación aparece como un elemento constitutivo, entre otros, de un lenguaje musical al que contribuye a caracterizar y dar forma. Más adelante, en el capítulo dedicado a la improvisación en el flamenco, Bailey $(1992,18)$ afirma que la improvisación idiomática no es una actividad separada y autónoma sino que siempre sirve a un cierto idioma, y es precisamente en a cumplir con el idioma a lo que se comprometen los improvisadores que trabajan en este ámbito. La improvisación no idiomática sería, en cambio, aquella en la que improvisar no tiene la función de representar una identidad idiomática, es decir, no pretende configurar, renovar o embellecer un idioma previo: en ella, improvisar es el núcleo, el motor principal de la música.

Esta categorización básica que abre la obra de Bailey resume a muy grandes rasgos una diferencia con la intención prospectiva de proveer al lector con herramientas que faciliten el entendimiento del texto. Si bien Bailey no repara en caracterizar con claridad la naturaleza de la aproximación no idiomática al acto de improvisar, podemos acceder a su entendimiento a través de los ejemplos que utiliza para describir su agencia.

La posibilidad y el sentido de la noción de improvisación no idiomática sigue siendo uno de los aspectos más debatidos entre practicantes y teóricos de las músicas improvisadas de diferentes generaciones. Bailey, introductor del concepto, se interesó más en especificar sus implicaciones y concreciones prácticas que en establecer una definición cerrada ya que, para él, todo intento de definir de manera tajante y cerrada la improvisación libre contraviene su naturaleza elusiva y por tanto implica, de alguna manera, una tergiversación de ésta (Bailey 1992, ix).

Fig. 2. Le Corbusier,"El Modulor" 


\section{NO-IDIOMATICIDAD: LA CUALIDAD DE LO NO IDIOMÁTICO}

"He utilizado los términos idiomática y no-idiomática para describir las dos formas principales de la improvisación. La improvisación idiomática, la más utilizada, se ocupa principalmente de la expresión de un idioma -tal como el jazz, el flamenco, la música barroca- y toma su identidad y motivación de dicho idioma. La improvisación no-idiomática tiene otros cometidos y se encuentra mayoritariamente en la llamada 'improvisación libre', y, si bien puede estar muy estilizada, generalmente no está unida a la representación de una identidad idiomática."

(Bailey 1992, xi-xii).

Una mirada cercana a estas palabras de Bailey puede ayudar a encontrar la clave para refundar una noción de improvisación no idiomática que vaya más allá de la concreción práctica que haya tenido en diferentes momentos históricos. Más que considerarla una categoría musical, el guitarrista de Sheffield, cuando dice que la improvisación no idiomática "se encuentra mayoritariamente en la llamada 'improvisación libre"' (xi), apunta hacia una cualidad posible, un estado potencial hacia el que la música improvisada puede tender. No en vano, en una entrevista realizada por Jean Martin en agosto de 1996, Bailey aclara que la mayor diferencia entre la improvisación libre y otras músicas improvisadas es que "éstas son idiomáticas mientras que la improvisación libre no lo es" (Bailey, 2003). A partir de las palabras de Bailey podemos plantear algunas de las preguntas más importantes que este artículo se plantea: a saber, ¿acaso la improvisación libre no sea susceptible de constituir también un cierto tipo de identidad idiomática?, ¿hasta qué punto esta manera de hacer música es ajena a procesos de asentamiento y tipificación normativa?, ¿qué ocurriría si esta música se idiomatizase?

Si bien en la literatura sobre improvisación percibimos una ubicua utilización del término improvisación no-idiomática en relación de sinonimia con la improvisación libre, las palabras de Bailey parecen más bien proponer una potencialidad, una tendencia no necesaria de la improvisación libre. Una cualidad posible que convendría nombrar como no-idiomaticidad. Abordarla de esta manera, no como un compartimento estanco sino como un estado posible, nos ayuda a mantener una distancia semántica crítica con la que identificar las tensiones dialécticas entre fuerzas y resistencias a los procesos de idiomatización y asentamiento, entendiendo cómo esta música no se ha librado de 
una cierta codificación pero reconociendo en ella, también, una fuerza reacia a toda estandarización.

\section{PROCESOS DE IDIOMATIZACIÓN EN LA MÚSICA LIBREMENTE IMPROVISADA}

\section{FREE MUSIC: LA ETAPA EMBRIONARIA}

Los polifiéticos orígenes de la improvisación libre se remontan a la primera mitad de la década de los años 60 , cuando ya algunos compositores de la tradición académica como Karlheinz Stockhausen comenzaban a experimentar con composiciones abiertas en las que el intérprete tenía libertad para la toma de ciertas decisiones (Beins 2011, 167). En 1964, Franco Evangelisti crea en Italia el Gruppo d'Improvisazione Nuova Consonanza, que si bien fue inicialmente un colectivo de compositores, contaba entre sus filas al pianista polaco-estadounidense Frederic Rzewski, que en 1966 fundaría Música Elettronica Viva, uno de los colectivos pioneros de la música libremente improvisada y protagonista de su etapa embrionaria. En el Reino Unido, por su parte, Derek Bailey había formado en 1963 junto a Gavin Bryars y Tony Oxley el Joseph Holbrooke Trio, que si bien comenzó tocando jazz convencional, sentó las bases para el desarrollo de la improvisación libre (Watson 2013, 51): para 1965, el trío ya se había liberado de toda influencia jazzística (Bailey 1992, 86) y sus conciertos consistían en improvisaciones completamente abiertas. No obstante, como señala Ben Watson, ya diez años antes, en 1953, Bailey solía juntarse con otros músicos para tocar sin acordar estructuras previas (Watson 2013, 35), creando una música que habría podido reconocerse como improvisación libre de haber tenido la experiencia, el lenguaje y la perspectiva necesaria para poder hacerlo. Los orígenes de este grupo encarnan como los de ningún otro el etos dialéctico de la improvisación libre desde sus inicios: tres músicos, descontentos con el establishment del jazz de su época en el Reino Unido, deciden juntarse para dar rienda suelta a su inquietud exploradora. Diferentes influencias son puestas en juego con la intención de ser superadas: desde la segunda escuela de Viena hasta el jazz de vanguardia, la New York School o la tradición europea académica de Darmstadt (Bailey 1992, 86): el resultado es una progresiva supresión de los lastres idiomáticos para dar paso a una música en continua lucha por librarse de su propia historia. También en 1963 la dramaturga, educadora y teórica teatral estadounidense 
Viola Spolin, como breviario de un proceso experimental que venía desarrollando desde los años cuarenta, publica su obra magna Improvisation for fhe Theater. A Handbook of Teaching and Directing Techniques (Spolin 1963), obra que influirá a los primeros improvisadoras libres. No mucho más tarde, en 1965, Eddie Prévost, Keith Rowe y Lou Gare, tres músicos con experiencia en diferentes formaciones de jazz en el Reino Unido, comienzan a juntarse semanalmente en una sala del Royal College of Art para saciar su inquietud y solucionar su descontento con el entorno musical que les rodea (Rey 2011, 211). Tras seis meses de intenso trabajo y con nuevas incorporaciones como el bajista Laurence Sheaff o el compositor, pianista y violoncellista Cornelius Cardew -quien, poco después, renegará de su inicial interés por la tradición electroacústica de Darmstadt (Cardew 2006, 149-227) interesándose por la escuela experimental de New York-, el colectivo había cumplido su propósito de eliminar de su música los elementos que aún se reconocían de los diferentes idiomas que sus integrantes habían trabajado -mayormente, el jazz-.

\section{LA IDIOMATIZACIÓN DE LA MÚSICA IMPROVISADA EN LOS AÑOS SETENTA Y OCHENTA}

Esta intensa actividad, no obstante, no durará mucho: durante la segunda mitad de los años 60 , muchos y muchas improvisadoras, habiéndose apaciguado el éxtasis inicial, comienzan a reivindicar sus diferencias, sus particularidades a la hora de entender la improvisación, y para el final de la década, la improvisación libre vive su primer repliegue. Al otro lado del Atlántico, la tradición americana y su actitud crítica con la improvisación europea (a través de la figura de John Cage, pero mediada también por la experiencia norteamericana de la improvisación en relación a la cultura afroamericana, cristalizada en el jazz) es una gran influencia para que algunos improvisadores empiecen a desencantarse con la naturaleza de su práctica. AMM comienza a sentir los achaques del proceso de asentamiento que sucede a las etapas de exploración. Hacia el final de la década de los años sesenta, varios de sus miembros militan en el Partido Comunista de Inglaterra Marxista-Leninista, alineado con Beijing, lo cual les mueve a replantearse cada faceta de su vida, incluyendo su práctica musical. Mientras que Eddie Prévost sigue creyendo en el potencial revolucionario de la improvisación libre, la facción más comprometida con la ortodoxia maoísta, compuesta por Rowe y Cardew, empieza a considerar esta práctica como desconectada de la necesidad imperante de emancipación de las masas populares. Así, para los años setenta, este colectivo comienza a disgregarse, presentándose en las actuaciones en directo como dos dúos independientes: por un lado Prévost y Gare, y por otro Rowe y Cardew (Pré- 
vost 1995, 21), quien también comenzará nuevas aventuras musicales con la Scratch Orchestra que fundó en el Morley College de Londres en 1969 junto a Howard Skempton y Michael Parsons (Rey 2011, 206) - y de la que posteriormente sería integrante John Tilbury - como experimento de acción política y social a través de la creación musical.

Por su parte, en 1966, cuando su música ya era claramente abstracta, el Joseph Holbrooke Trio da por terminado su proceso experimental y se disuelve: Bryars, el díscolo del grupo, cada vez más crítico con la improvisación libre, se centra en su trabajo compositivo de tradición cageana (Watson 2013, 102); Oxley y Bailey emprenden caminos separados. Bailey se mudará a Londres y comenzará a frecuentar las sesiones de improvisación del Little Theatre Club, caldo de cultivo del Spontaneous Music Ensemble liderado por John Stevens, el primer músico británico en organizar un taller de música improvisada con periodicidad regular (Bailey 1992, 118). Allí fundará la Musical Improvisation Company en 1968 con Evan Parker, Hugh Davies, Jamie Muir -que abandonó la formación en 1972 para formar parte del grupo de rock progresivo King Crimson (202)- y Christine Jeffrey (94).

Tras el abandono de la Musical Improvisation Company por parte de Miur al comienzo de la nueva década, Bailey empezará a centrarse en su trabajo en solitario. De hecho, el interés por la improvisación en solitario se cristalizará en un nuevo proyecto que no sólo encarnará su entendimiento de la música improvisada sino que será su particular respuesta al enquistamiento cada vez más latente que percibía en estas músicas: las Company Weeks, organizadas por el músico británico desde 1977 hasta 1991, en las que músicos que nunca habían tocado juntos antes colaboraban a través de la improvisación. Esta conjunción esporádica personalidades dispares nació como estrategia dialéctica para confrontar el asentamiento de las músicas improvisadas. Comparándolos con una aventura amorosa, para Bailey estos encuentros son "episodios cortos e intensos que afectan a los músicos y las audiencias de por vida" (Watson 2013, 214). No obstante, el filósofo y crítico musical Andy Hamilton, al respecto de esta actitud inconformista característica de Derek Bailey, nos recuerda que este guitarrista británico, más allá de toda reivindicación no idiomática, desarrolló una manera de improvisar inconfundible que lo convierte en un improvisador idiomático (Hamilton 2000, 182). La misma actitud crítica hacia este posicionamiento lo percibimos en las palabras del percusionista británico Eddie Prévost, miembro fundador de AMM, cuando dice que sin rasgos idiomáticos, la manera de tocar de Derek Bailey no sería tan reconocible (Prévost 2004, 14). 
Este acercamiento individualista propio del pensamiento baileyano puede entenderse como idiomático en tanto que enfatiza la investigación y la búsqueda del idioma propio del sujeto, la configuración de un repertorio de técnicas reconocibles y asociadas a un determinado instrumentista. Al respecto de ello, teóricos de la improvisación no idiomática como el improvisador francés Jean-Luc Guionnet postulan que, en tanto que el improvisador trabaja en tiempo real, ante lo incierto, lo importante no es tanto la adquisición de unas destrezas técnicas características del sujeto sino la manera que éste las pone en juego en la improvisación. Esta visión se evidencia en su idea de libertad sin menú: la posibilidad de utilizar los recursos de los que el instrumentista dispone, pero sin considerar una serie de opciones previas (Guionnet, 2014, comunicación personal). Frente a la postura baileyana que enfatiza el contraste entre la personalidad musical de dos o más músicos en el acto de improvisar, se sitúa también la visión heurística propuesta por Eddie Prévost según la cual, más que limitarse a una serie de registros previamente determinados, el aspecto más importante del trabajo del improvisador consiste en el descubrimiento de su propio sonido en un proceso investigativo que ocurre en la propia performance, trabajando con los materiales en tiempo real y siempre en función de la acción de los otros músicos y a los sonidos fortuitos que puedan ocurrir en el espacio. En palabras del propio Prévost, este impulso heurístico es "una determinación para descubrir la naturaleza de las cosas" (Prévost 2004, 16).

Es importante recordar que el surgimiento de la improvisación libre coincide cronológicamente con la intensificación de la guerra de Vietnam y su respuesta en el auge de la contracultura; la popularidad de las teorías maoístas -que influirán ampliamente a muchos improvisadores británicos- como consecuencia de la debilitación del paradigma soviético, y el auge del post-marxismo. Un convulso panorama político en el que también se ven implicados los protagonistas de los primeros episodios de estas prácticas musicales. Los códigos éticos implícitos en la aproximación comunal a la práctica de la improvisación propios de AMM o Musica Elettronica Viva (Alonso 2008, 38) contrastan con el énfasis individualista de la escuela baileyana. No obstante, si bien los encuentros esporádicos de improvisación propuestos por Derek Bailey se entendían como una resistencia a la idiomatización y asentamiento de los lenguajes improvisatorios que afectaba a las las formaciones musicales cerradas y estables, las Company Weeks fundan una tradición de improvisación ad-hoc que se extiende hasta nuestros días y podemos identificar en la música que se presenta en las series de conciertos Mopomoso en Londres - eventos organizados por el guitarrista post-baileyano John Russell. En esta tradición, la música parece convertirse en un subproducto de las relaciones 
sociales, y el acto de improvisar, en un laboratorio en el que experimentar nuevas configuraciones para la interacción humana. Como reconocen músicos como Seijiro Murayama, si bien Bailey representó originariamente el espíritu de lo no-idiomático, su legado no tarda en convertirse en dogma y normatividad (Murayama, 2013), como parte de lo que en la literatura especializada ha sido denominado E.F.I., European Free Improvisation. Así, la libertad propia de la noción de improvisación libre -que, siguiendo a Peters, sería una libertad negativa: libre de los condicionamientos más que libre para actuar autónomamente (Peters 2009, 141)-, paradójicamente, comienza a levantar imaginarios y prefigurar no sólo maneras concretas de abordar el acto de improvisar sino, más específicamente, de cómo la música improvisada debe sonar. La cristalización de este paradigma tendrá su consecuencia más extrema en la estética denominada jocosamente como 'plinky plonk' o 'skritchy scratch' (Davies 2011, 70), que estará en auge durante los años ochenta: una lógica conversacional, idiomática, casi análoga discurso humano, relacionada con la idea del concepto vocal del orígen de la música fundada por el musicólogo berlinés Curt Sachs (Sachs 1977, 110-1): la música como aserción individual y celebración de la subjetividad. Contra este acercamiento individualista procede recordar a la contrabajista portuguesa Margarida Garcia cuando afirma que, en una improvisación colectiva, hay que superar la individualidad y conformar una identidad colectiva en la que los instrumentistas actúen "como si cada uno fuera la tecla de un piano" (García, comunicación personal, 2010).

\section{La nUEVA improvisación en los años nOVEnta: Onkyo, NeW LONDON Silence, Echtzeitmusik}

En los años noventa, esta manera ya clásica de improvisar empieza a presentar síntomas de debilidad. De manera sincrónica pero no interconectada, en países tan dispares como Japón, Alemania, Austria o el Reino Unido (a pesar de que en Londres aún estaba muy arraigada la herencia baileyana) se empiezan a generar respuestas y proponer alternativas al agotamiento de unos lenguajes que ya no responden a las inquietudes creativas de los improvisadores más jóvenes (Davies 2011, 69). El Onkyo en Tokyo, Echtzeitmusik en Berlín, New London Silence en Londres o el trombonista austriaco Radu Malfatti (cuyo particular viraje estilístico comenzó en el free jazz en los años sesenta y terminó en las vertientes más silenciosas de la improvisación para finales de los años noventa, habiendo abandonado actualmente la música improvisada) en Viena, desarrollan una nueva estética que no sólo introduce nuevos parámetros como la relación entre el sonido intencional y accidental y la sensibilidad hacia el sonido de los espacios en donde se improvisa (Kammerer 2011, 94), 
sino, mucho más importante, coloca el acto de improvisar en la esencia misma de la música, el motor que la pone en marcha: frente a la idea baileyana en la que la música es casi un pretexto para ejercitar la improvisación, para esta nueva generación, la improvisación será simplemente un desarrollo técnico con el que llegar a la música. Esto nos recuerda al planteamiento del percusionista japonés Seijiro Murayama, al hablar de este cambio de paradigmas en relación con la posibilidad de una improvisación no idiomática, cuando señala que hay que trabajar "de los sonidos hacia la música" (Murayama 2013), y no en sentido contrario, partiendo de una idea predeterminada y clasificable de música hacia donde dirigir los sonidos.

\section{ECHTZEITMUSiK: MÚSicA EN TIEMPO REAL}

En Berlín, ya desde antes de la caída del Muro, el free jazz -aceptado y promovido por el gobierno de la D.D.R. por su supuesta filiación política confrontacional y anticapitalista-, con formaciones como el Zentralquartett de Conny Bauer, tenía una influencia tal en el panorama musical que incluso repercutía en las prácticas musicales libremente improvisadas (Neumann 2011, 209), actuando como un lastre en su vocación renovadora y experimental, abocándola a sus formas más primitivas. Éstas eran aún estimadas por la audiencia en espacios autogestionados como Anorak, donde los primeros conciertos de esta nueva estética de la improvisación tenían lugar (Blažanović 2011, 29). La sensación de descontento de los más jóvenes genera un examen exhaustivo de las asunciones y prejuicios heredados respecto a la naturaleza y especificidad de la improvisación musical libre, una criba de las ideas aprehendidas que habían constituido hasta ese momento el imaginario propio de la música improvisada: aspectos como interacción, individualidad, espontaneidad o libertad y su papel en el acto de improvisar, comienzan a ser sometidas a juicio crítico. Uno de los aspectos que rebela su interés por liberar a la improvisación musical de los lastres idiomáticos que percibían en la tradición de la Libre Improvisación Europea era su insistencia en desvincularse de lo libre, del free, que percibían todavía como una atadura histórica con el free jazz. En cierto modo, una adjetivación de la improvisación, una cualificación que la idiomatizaba. En palabras del músico austriaco Christoff Kurzmann, "la gente no quería ser improvisadora. [...] Nosotros no éramos jazz, éramos simplemente 'improv.'" (Kurzmann 2011, 348). Estos jóvenes músicos comienzan a utilizar el término Echtzeitmusik, 'música en tiempo real', para referirse a su práctica. Este nombre se adopta con la intención de separarse de otras tradiciones de improvisación que ellos percibían manidas e idiomatizadas: tanto el free jazz como la improvisación libre de tradición europea (Blažanović 2011, 39). A dife- 
rencia de éstas, Echtzeitmusik rechaza la idea de la improvisación como una categoría cerrada y propone un acercamiento polimorfo y ambiguo, una multitud de aproximaciones mixtas cuyo aspecto común es el trabajo con el material en tiempo real, un énfasis que resta importancia a la división entre improvisación y composición: según la académica serbia Marta Blažanović, este manto social ecléctico y plurifocal que se generó en Berlín durante los últimos años de la década de los noventa con el surgimiento de Echtzeitmusik tendió puentes entre compositores e improvisadores, descubriendo las posibilidades de la composición a muchos improvisadores y despertando en muchos compositores el interés por la improvisación (Blažanović 2012, 63-4). El epítome de esta nueva manera de entender el acto de improvisar se encuentra en el forjamiento de la noción de composer-performer, una noción que hace justicia a la complejidad y gran variedad de acercamientos por parte de muchos músicos cuyo trabajo no se puede clasificar dentro de las categorías tradicionales de composición e improvisación: este término designa al músico que, aún dejando un ancho margen para lo imprevisto, la toma y el cambio de decisiones en tiempo real, hace uso de ciertas anotaciones escritas, diseñando una hoja de ruta con la intención de llegar a resultados sonoros que no ocurrirían espontáneamente en una situación totalmente improvisada (Streich 2011, 131). La naturaleza de estas anotaciones -en cierto modo un tipo de escritura musical- también es entendida de manera particular: el composer-performer del entorno Echtzeitmusik generalmente utiliza la notación como una herramienta mnemotécnica para propiciar el surgimiento de situaciones sonoras que no serían posibles de otro modo, esto es, como un recurso técnico para guiar la situación en tiempo real y no con la idea de configurar una composición fija que pueda ser interpretada por otros músicos (Neumann 2011, 131).

Aunque para algunos músicos improvisadores del entorno berlinés de Echtzeitmusik como el sueco Sven-Åke Johansson esta manera de improvisar no configura de manera alguna un lenguaje (Johansson 2011, 115), lo cierto es que las prácticas de estos improvisadores no estaban exentas del riesgo de configurar nuevos modelos normativos. Si bien a día de hoy la música improvisada de Berlín, heredera de la generación Echtzeitmusik, no constituye una identidad idiomática uniforme, sí se puede reconocer la recurrencia, en varios improvisadores, de ciertos rasgos estilísticos que han configurado lo que hoy se conoce como el sonido Berlín (Blažanović 2012, 65): una manera concreta de improvisar caracterizada por el uso de materiales musicales de naturaleza relativamente simple, tales como ruidos blancos, estructurados en largos tramos de sonido y silencio. Al fin y al cabo, un estilo al que, de hecho, muchos músicos de todo el mundo, sin arraigo ni experiencia de la evolución de estas 
prácticas musicales en la capital alemana, parecen amoldarse cuando van allí a tocar.

\section{REDUCCIONISMO}

El musicólogo alemán Peter Niklas Wilson nos recuerda que, en la improvisación libre, el desarrollo de la personalidad instrumental está tan valorado que cualquier adaptación de los recursos de otro músico sería "casi un sacrilegio" (Wilson 1999, 17). También otros como Mattin Artiach, Ray Brassier, Jean-Luc Guionnet y Seijiro Murayama señalan que la improvisación ensalza algunas de las características en alza en la economía capitalista, tales como la autopromoción y la individualidad, bajo la lógica del nicho de oportunidad: "lo que yo ofrezco no se encuentra en ninguna otra parte" (Artiach et al. 2010, 16).

Si bien la escena Echtzeitmusik surge como reacción al estancamiento del lenguaje de la improvisación libre a finales de los años noventa, ésta también ha ido sufriendo el efecto de la normalización. Axel Dörner, uno de los protagonistas musicales de su generación, cuya obra es clave para entender la respuesta a la idiomatización de la música improvisada que Echtzeitmusik supuso en los años noventa, es hoy en día uno de los ejemplos más evidentes: reinventando la trompeta y extrayendo de ella sonoridades no exploradas hasta entonces, desarrolló un lenguaje puramente reconocible y personal que sentó las bases de la estética reduccionista.

El reduccionismo, surgido en los primeros años de la década de los dos mil, es una de las consecuencias más remarcables y al mismo tiempo paradójicas del cambio de paradigmas que afectó desde mediados de los años noventa a las prácticas musicales improvisadas en Europa y Asia. Músicos como Robin Hayward, Andrea Neumann o Axel Dörner fundaron una manera de improvisar que eliminaba deliberadamente toda reminiscencia gestual y toda interacción dialógica -la cual consideraban idiomática-, centrándose en parámetros como la estructura temporal del discurso musical o la integración de las particularidades aurales de los espacios en el flujo de la música, adaptando sus dinámicas al sonido del entorno de manera que las contingencias sonoras del mundo pasan a primer plano. Una posible definición de esta estética es la aportada por el trompetista inglés Matt Davies: para él, el reduccionismo consiste en "reducir información, concentrándose en el mundo que habita y las relaciones que crea" (Davis 2005, 174). 
La supuesta radicalidad de esta música ha sido cuestionada por varios practicantes y teóricos de la música improvisada. Eddie Prévost, miembro de AMM, acusa a esta estética de formalista y de estar desligada de las implicaciones sociales de la investigación estética, siendo así contraria al etos dialéctico de la improvisación e intentando imponer en la audiencia una "pesimista religiosidad" (Prévost 2004, 38). No en vano una de las consecuencias previsibles de este proceso de idiomatización de las prácticas improvisatorias en Berlín, a medida que éstas fueron derivando en una manera de tocar cada vez más estricta, normativizada y predeterminada por el compromiso con un código estético previo al acto de hacer música, fue el abandono de la improvisación por parte de muchos músicos. El clarinetista argentino Lucio Capece, uno de los herederos contemporáneos de la estética reduccionista, afirma que para dejar su idioma, tuvo que dejar de improvisar (Capece, comunicación personal, 2014). Esto nos recuerda inevitablemente la crítica cageana a la improvisación, según la cual ésta condena a la música a los límites de la subjetividad marcados por el aprendizaje y el gusto personal (Turner 1992, 472), contra lo cual el proceso experimental propone procedimientos de azar y aleatoriedad.

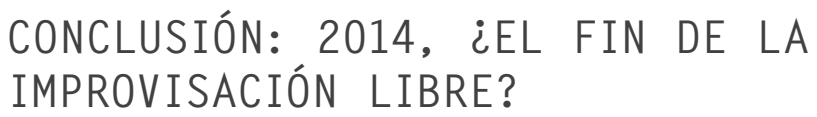

En febrero de 2014, el músico británico Richard Thomas vaticinaba, en la revista The Wire, culmen del establishment anglosajón de la música experimental, la muerte de la improvisación libre no idiomática (Thomas, 2014). Sin lugar a dudas, una llamada de atención para todas las personas comprometidas con esta manera de hacer música que son conscientes de su situación de estancamiento actual. Este artículo ha procurado sentar las bases de un entendimiento de lo no idiomático como un posicionamiento crítico y resistente a los procesos de normalización que se ha mantenido en las diferentes generaciones de músicos improvisadores. Reconocer la recurrencia, en el núcleo de esta práctica, de una fuerza resistente a su cosificación, nos ayuda a recuperar el sentido real del potencial no idiomático que Bailey reconoció en la improvisación libre -una música resistente a su enquistamiento (Bailey 2004, 13) - reinaugurando la posibilidad de intuir el umbral que da lugar a aquello que ésta no es. La posibilidad de recuperar su ruido. 


\section{Referencias}

Alonso, Chefa. 2008. Improvisación libre: La composición en movimiento. Baiona: Dos Acordes

Artiach Oraa, Mattin, Ray Brassier, Jean-Luc Guionnet \& Seijiro Murayama. 2010. Idioms and Idiots, booklet de acompañamiento del CD-ROM.Estocolmo: w.m.o/r

Bailey, Derek. 1992. Improvisation: Its Nature and Practice in Music. New York: Da Capo

- 1996. "Derek Bailey Interview, 16 August 1996: Interview copyright Jean Martin". http:// www.efi.group.shef.ac.uk/fulltext/mbailin2.html

Beins, Burkhard. 2011. "Scheme and Event". En Echtzeitmusik Berlin: Selbstbestimmung einer Szene [Selfdefining a scene], editado por Burkhard Beins, Christian Kesten, Gisela Nauck \& Andrea Neumann, 167-79. Hofheim: Wolke

Blažanović, Marta. 2011. "Social History of the Echtzeitmusik Scene in Berlin". En Echtzeitmusik Berlin: Selbstbestimmung einer Szene [Selfdefining a scene], editado por Burkhard Beins, Christian Kesten, Gisela Nauck \& Andrea Neumann, 29-52. Hofheim: Wolke

Blažanović, Marta. 2012. "Echtzeitmusik: The social and discursive contexts of a contemporary music scene". Tesis Univ. Humboldt (Berlin)

Cage, John. 1990. “John Cage's Practical Utopias - John Cage in Conversation with Steve Sweeney Turner” en The Musical Times 131(1771):469. DOI: 10.2307/1193658

Cardew, Cornelius. (1974) 2006. "Stockhausen Serves Imperialism". En Cornelius Cardew (1936-1981): A Reader, editado por Edwin Prévost, 149-227. Essex: Copula. Publicado por primera vez por Latimer New Dimensions

Davies, Rhodri. 2011. "Berlin-London 1997-1999". ". En Echtzeitmusik Berlin: Selbstbestimmung einer Szene [Selfdefining a scene], editado por Burkhard Beins, Christian Kesten, Gisela Nauck \& Andrea Neumann, 67-77. Hofheim: Wolke

Davis, Matt. "Trumpet, electronics, field recordings". En Blocks of Consciousness and the Unbroken Continuum, editado por Brian Marley y Mark Wastell. Londres: Continuum

Hamilton, Andy. 2000. "The Art of Improvisation and the Aesthetics of Imperfection". British Journal of Aesthetics 40(1): 168-85

Kammerer, Margareth. 2011. "Echtzeitmusik" En Echtzeitmusik Berlin: Selbstbestimmung einer Szene [Selfdefining a scene], editado por Burkhard Beins, Christian Kesten, Gisela Nauck \& Andrea Neumann, 93-4. Hofheim: Wolke

Kurzmann, Christof. 2011. "Jericho and Silicon Valley. Johannes Bauer and Christof Kurzmann in Conversation with Tobias Herold and Marta Blažanović". En Echtzeitmusik Berlin: Selbstbestimmung einer Szene [Selfdefining a scene], editado por Burkhard Beins, Christian Kesten, Gisela Nauck \& Andrea Neumann, 337--58. Hofheim: Wolke

Mattin [Mattin Artiach Oraa], Ray Brassier, Jean-Luc Guionnet \& Seijiro Murayama. 2010. Idioms and Idiots, booklet de acompañamiento del CD-ROM. Estocolmo: w.m.o/r

Murayama, Seijiro. 2013. "Elkarrizketa [Entrevista]: Seijiro Murayama". Invitado del taller Rastros: Improvisación Libre y Construcción junto a Itziar Okariz en Arteleku (Donostia-San Sebastián, 2-7 abril). Vídeo de Vimeo, 26:36. Publicado 11 abril. http://vimeo. com/63815680

Neumann, Andrea. 2011. "Playing Inside Piano". En Echtzeitmusik Berlin: Selbstbestimmung einer Szene [Selfdefining a scene], editado por Burkhard Beins, Christian Kesten, Gisela Nauck \& Andrea Neumann, 202-13. Hofheim: Wolke 
Peters, Gary. 2009. The Philosophy of Improvisation. Chicago: University of Chicago

Prévost, Edwin. 1995. No Sound is Innocent. Matching Tye, UK: Copula / Matchless Recordings

- 2004. Minute Particulars. Matching Tye, UK: Copula / Matchless Recordings

Rey Vizcaíno, Héctor. 2011. "Música Experimental en el País Vasco: el eje Bilbo-Donostia-Bera". Trabajo fin de máster Univ. del País Vasco

Sachs, Curt. 1977. The Wellsprings of Music. New York: Da Capo

Spolin, Viola. 1963. Improvisation for the Theater: A Handbook of Teaching and Directing Techniques. Evanston: Northwestern University

Streich, Stefan. 2011. "Labor Diskurs". En Echtzeitmusik Berlin: Selbstbestimmung einer Szene [Selfdefining a scene], editado por Burkhard Beins, Christian Kesten, Gisela Nauck \& Andrea Neumann, 123-45. Hofheim: Wolke

Thomas, Richard. 2014. "The Symptom Is Death". Versión electronica de The Wire, In Writing /Columns, February 2014. Acceso 20 sept. 2015. http://www.thewire.co.uk/in-writing/ columns/richard-thomas_the-symptom-is-death

Trio Sowari (Phil Durrant, Bertrand Denzler Burkhard Beins). 2011. "27 Questions... and some possible answers". En Echtzeitmusik Berlin: Selbstbestimmung einer Szene [Selfdefining a scene], editado por Burkhard Beins, Christian Kesten, Gisela Nauck \& Andrea Neumann, 113. Hofheim: Wolke

Watson, Ben. 2013. Derek Bailey and the Story of Free Improvisation. Londres: Verso

Wilson, Peter Niklas. 1999. Hear and Now: Gedanken zur improvisierten Musik. Hofheim: Wolke 